\title{
ACHILLES TENOTOMY PERFORMED PERCUTANEOUSLY UNDER LOCAL ANESTHESIA IN OPERATION THEATER ROOM IN CLUBFOOT.
}

1. MBBS, FCPS (Ortho) Assistant Professor

Department of Pediatric Orthopedics, The Children's Hospital and The Institute of Child Health, Lahore.

2. MBBS, MS

Professor

HOD

Department of Pediatric Orthopedics The Children's Hospital and

The Institute of Child Health, Lahore.

3. MBBS, FCPS (Ortho)

Senior Registrar

Department of Pediatric Orthopedics

The Children's Hospital and

The Institute of Child Health, Lahore.

Correspondence Address:

Dr. Farhad Alam

House No. P324, Galli No.3,

Alnajaf Colony, Faisalabad.

drfarhadgilgity@yahoo.com

Article received on:

22/02/2019

Accepted for publication:

15/05/2019

Received after proof reading:

$28 / 08 / 2019$

\section{Abdul Latif Shahid ${ }^{1}$, Abdul Latif Sami², Farhad Alam ${ }^{3}$}

ABSTRACT... Objectives: Achilles tenotomy is required in $80 \%$ of cases after the Ponseti method in clubfoot. There are many complications reported if it is perfomed percutaneously under local anesthesia in the clinic. Complications are bleeding from either posterior tibial artery or peroneal artery and sometimes from small saphenous vein. Nerve injuries like tibial nerve and sural nerve are also documented. Incomplete Achilles tenotomy is another complication responsible for recurrence of deformity and redo tenotomy. On the other hand, mini-open Achilles tenotomies performed under general anesthesia in Operation Theater have no such complications as mentioned above. The rationale of this present study is to document the safety and any complications when Achilles tenotomy is performed percutaneously under local anesthesia instead of mini-open technique under general anesthesia in the operation theater room. Study Design: Retrospective review study. Setting: Pediatric Orthopedic Department of the Children Hospital and the Institute of Child Health, Lahore. Period: 2014 to 2018. Material and Methods: In infants with congenital talipes equinovarus who underwent percutaneous Achilles tenotomy in operation theater room under local anesthesia. Results: Fifty patients with seventy five feet included in this retrospective study who underwent Achilles tenotomy percutaneously under local anesthesia in operation theater room. Before starting Ponseti casts, average Pirani score was 5.6.Before Achilles tenotomy, the average number of casts applied was 4.9.11.9 weeks was the average age at the time of Achilles tenotomy. No vascular, neural and tendon related complications occured in any infants and they discharged from hospital on same day. Conclusions: Achilles tenotomy performed percutaneously under local anesthesia in the operation theater room is more safe and comfortable for both family and pediatric orthopedic surgeon in clubfoot. No vascular, neural and tendon related complications occurred. Operation theater environment and local anesthesia are very beneficial for eliminating any chance of vascular, neural and tendon related complications.

Key words: $\quad$ Achilles Tenotomy, Clubfoot, Percutaneous.

Article Citation: Shahid AL, Sami AL, Alam F. Achilles tenotomy performed percutaneously under local anesthesia in operation theater room in clubfoot. Professional Med J 2019; 26(9):1477-1481. DOI: 10.29309/TPMJ/2019.26.09.3312

\section{INTRODUCTION}

The Ponseti method is a universally accepted, keenly learned and mostly adopted method for the treatment of idiopathic clubfoot. Dr. Ignacio pioneered this method in 1940. In this method, clubfoot is corrected by serial specific manipulations and then application of long-leg, well-molded plaster casts on the weekly basis to correct midfoot cavus, forefoot adduction, and subtalar varus components of the deformity. ${ }^{1}$ Finally, Achilles tenotomy is performed for equinus deformity correction in approximately $80 \%$ cases. $^{2}$ This method is beneficial for infants because of cartilaginous nature of their bony structures, and their ligaments, tendons, muscles and joint capsules well respond to stretching and plaster casts. $^{3}$

Achilles tenotomy is performed percutanously under local anesthesia in surgeon office. Botulinum toxin injection into the calf muscles ${ }^{4}$, percutaneous needle sectioning of Achilles tendon $^{5}$ and a mini-open technique for Achilles tenotomy ${ }^{6}$ are different modifications in this usual procedure. Similarly it can be performed in operation theater or in outpatient clinic under general anesthesia ${ }^{7}$, under propofol sedation ${ }^{8}$ or under topical anesthesia. 
When Tendoachilles temnotomy is performed percutaneously in the clinic, many complications are reported like bleeding from either posterior tibial artery or peroneal artery and sometimes from small saphenous vein. ${ }^{9}$ Nerve injuries like tibial nerve and sural nerve are also documented. ${ }^{10}$ Incomplete release of Tendoachilles is another complication responsible for recurrence of deformity. ${ }^{11}$

The rationale of this present study is to document the safety and any complications when Tendoachilles tenotomy is performed percutaneously under local anesthesia in the operation theater room instead of mini-open technique under general anesthesia.

\section{MATERIALS AND METHODS}

This was a retrospective study performed from 2014 to 2018 in Pediatric Orthopedic Department of the Children Hospital and the Institute of Child Health, Lahore. Total fifty children with seventy five feet were included in this study. Our inclusion criteria was only idiopathic clubfoot not taking any treatment. All children with syndromic clubfoot, one foot having clubfoot while other foot has any other anomaly and children already taking treatment from somewhere else were excluded from the study. All children underwent standard Ponseti technique casts in our Ponseti clinic before Achilles tenotomy. When all other deformities except equinus were corrected, the children underwent percutaneous Achilles tenotomy under local anesthesia in operation theather room. Following data of each patient was recorded: gender, age at start of treatment, Pirani score at start of treatment, age at tenotomy, unilateral or bilateral foot involvement, number of casts required before tenotomy, vascular or nerve injury and need for redo tenotomy.

The procedure was performed in a dedicated room in operation theater. All tenotomies were done by senior auther himself with a resident doctor alongwith one male paramedical staff and one nurse. Resident doctor, male paramedical staff and nurse were deputed only in this room for this specific procedure. Before doing percutaneous Achilles tenotomy it was assessed by senior author that all other deformities were corrected and children only required equinous correction. After proper sterilization and draping Achilles tendon was palpated by maximally dorsiflexing the foot. Only $0.5 \mathrm{ml}$ local anesthetic injection was given so that Achilles tendon remained papable for proper and complete sectioning. After one minute of injection, again Achilles tendon was palpated and when sure it was sanctioned $1 \mathrm{~cm}$ above the insertion of Achilles tendon. Tenotomy was confirmed by increase in dorsiflexion upto 15 degree above neutral. Tenotomy site was compressed by gauzepieces for one minute. The final cast was applied by maximally dorsiflexing the foot along with full abduction for three weeks.

Any complication immediate or late were documented. After three weeks, cast was removed and foot assessed for correction. Now patient order to wear foot abduction brace and called every month for follow-up.

\section{RESULTS}

Fifty patients with seventy five feet involved in this study underwent Achilles tenotomy percutaneously in operation theater room. Twenty five children had bilateral, fifteen had right-sided and ten had left-sided tenotomy. Before starting Ponseti casts, average Pirani score was 5.6.Before Achilles tenotomy, the average number of casts applied was 4.9.11.9 weeks was the average age at the time of Achilles tenotomy.

No complaint gave by parents regarding plaster problems or pain or loss of sleep or excessive crying.

No complications regarding bleeding from posterior tibial artery or peroneal artery or small saphenous vein occurred. No tibial or sural nerve injury was documented. No child needed redo tenotomy.

All tenotomies wound healed after removal of cast. After cast removal, dorsiflexion was as maximum as before cast application and without any discomfort or pain.

Two children developed minor problems which 
dealt immediately and proprly. One child came with wetting of cast accidently by falling in basket containing water. Cast immediately changed after thorough care of tenotomy wound. Another chilld came with removal of cast after two weeks. On further inquiry, parents told that cast was required only for two weeks according to their understanding. This child again applied cast for one week more to complete three weeks which is needed for complete healing of Tendoachilles tendon.

Another child developed a major problem after one year of follow-up and that was dynamic supination during walking. He was again manipulated and applied cast but finally needed tibialis anterior tendon transfer.

\section{DISCUSSION}

The idiopathic clubfoot is now treated by Ponseti method all over the world. Treatment should be started as early as possible. In the Ponseti technique, serial manipulatin of foot is done at one week interval and this specific position of foot is then maintained by well-moulded long leg cast. ${ }^{1}$ First cavus is corrected, then adduction and varus are corrected and finally equinus is correct. All deformities are corrected by manipulation and casting except equinus which is corrected by Achilles tenotomy and casting. ${ }^{2}$ Another difference is that all plasters are changed at weekly interval except last one which is changed after three weeks for complete healing of Tendoachilles tendon. After this correction phase, maintenance phase is stated by wearing foot abduction brace 23 hours a day for three months and then during night and laps time upto four years.

Achilles tenotomy is usually performend percutaneously under local anesthesia in surgeon office. Now there are many modifications to this usual procedure regarding place of procedue, type of anesthesia and procedure technique. Each modification has advantages and disadvantages. Botulinum toxin injection into the calf muscles ${ }^{4}$, percutaneous needle sectioning of Achilles tendon ${ }^{5}$ and a mini-open technique for Achilles tenotomy ${ }^{6}$ are modifications in procedure technique .Similarly it can be performed in operation theater and in outpatient department. This is modification in procedure place. Last modification is type of anesthesia which include general anesthesia ${ }^{7}$, propofol sedation $^{8}$ and topical anesthesia.

Achilles tenotomy by mini-open technique reduces the chances of incomplete tenotomy and neurovascular injury by direct visualization of structures in the operating room but cost of operation theater and complications of general anesthesia are risk factors. ${ }^{12}$

Percutaneous Achilles tenotomy with a wide gauge needle under local anesthesia has less chances of complications as compared to a surgical blade, less invasive, more safe, simple and effective as compared to other methods of tenotomy. ${ }^{13}$

Percutaneous Achilles tenotomy performed under local anesthesia in the office is easy, simple, low cost but many complications reported in literature. These complications are bleeding from either posterior tibial artery or peroneal artery and sometimes from small saphenous vein ${ }^{9}$, nerve injuries like tibial nerve and sural nerve ${ }^{10}$ and incomplete release of Tendoachilles. ${ }^{11}$

Robert Jay in his study showed that Botulinum A toxin is not helpful in decreasing correction time for casting and eliminating need for percutaneous Achilles tenotomy. ${ }^{4}$

In this study we performed Achilles tenotomy both percutaneously and under local anesthesia but in operation theater. The only difference is place of procedure. This is the first study done in this regard and has many advantages. Good procedure control by surgeon, more safety, better handling of complications, more chances of training of residents and availability of anesthetists are some of these advantages.

Percutaneous Achilles tenotomy performed in the office or outdoor clinic has many complications but we have no complication in our study. Cost is also comparable with office or clinic procedure because we only use operation theater not 
general anesthesia or sedation. In one way our study is similar in reducing complications while on the other way is superior in cost and safety issues to mini-open technique of Achilles tenotomy performed in operation theater under general anesthesia.

This technique is more useful and acceptable because we are doing tenotomy without general anesthesia in operation theater. There are always risks for infants in general anesthesia. DiMaggio et al. in their study showed that children have behavioral and developmental disorders with early exposure to anesthesia. ${ }^{14}$

\section{CONCLUSION}

Achilles tenotomy performed percutaneously and under local anesthesia in the operation theater is more safe and effective than another method of Achilles tenotomy. There are no complications because of operation theater environment. Cost and time consumption is similar to office or clinic procedure. Hence, this technique can be adopted in place of mini-open technique of Achilles tenotomy performed under general anesthesia. Copyright@ 15 May, 2019.

\section{REFERENCES}

1. van Bosse HJ. Ponseti treatment for clubfeet: An international perspective. Curr Opin Pediatr. 2011; 23:41-45.

2. Scher DM, Feldman DS, van Bosse HJ, et al. Predicting the need for tenotomy in the Ponseti method for correction of clubfeet. J Pediatr Orthop. 2004; 24:34952.

3. Ponseti IV, Smoley EN. The classic: congenital club foot: The results of treatment. 1963. Clin Orthop Relat Res. 2009; 467:1133-45.

4. Cummings RJ. The effectiveness of botulinum A toxin as an adjunct to the treatment of clubfeet by the Ponseti method: A randomized, double blind, placebo controlled study. J Pediatr Orthop. 2009; 29: 564-69.
5. Minkowitz B, Finkelstein BI, Bleicher M. Percutaneous tendo Achilles lengthening with a large-gauge needle: A modification of the Ponseti technique for correction of idiopathic clubfoot. J Foot Ankle Surg. 2004; 43:263-65.

6. Dogan A, Kalender AM, Seramet E, et al. Mini-open technique for the achilles tenotomy in correction of idiopathic clubfoot: A report of 25 cases. J Am Podiatr Med Assoc. 2008; 98:414-17.

7. Parada SA, Baird GO, Auffant RA, et al. Safety of percutaneous tendo achilles tenotomy performed under general anesthesia on infants with idiopathic clubfoot. J Pediatr Orthop. 2009; 29:916-19.

8. Bor N, Katz $\mathrm{Y}$, Vofsi $\mathrm{O}$, et al. Sedation protocols for ponseti clubfoot achilles tenotomy. J Child Orthop. 2007; 1:333-35.

9. Dobbs MB, Gordon JE, Walton T, Schoenecker PL (2004) Bleeding complications following percutaneous tendoachilles tenotomy in the treatment of clubfoot deformity. J Pediatr Orthop 24:353-57.

10. Changulani M, Garg N, Bruce CE (2007) Neurovascular complications following percutaneous tendoachillis tenotomy for congenital idiopathic clubfoot. Arch Orthop Trauma Surg 127:429-30.

11. Jowett CR, Morcuende JA, Ramachandran M (2011) Management of congenital talipes equinovarus using the Ponseti method: a systematic review. J Bone Joint Surg Br 93(9):1160-64.

12. Rhett $M$, William $H$, Brian $S$, Garrett L. A mini-open technique for Achilles tenotomy in infants with clubfoot. J Child Orthop. 2016; 10(1):19-23.

13. Minkowitz B, Finkelstein $\mathrm{Bl}$, Bleicher M. Percutaneous tendoAchilles lengthening with a large-gauge needle: A modification of the Ponseti technique for correction of idiopathic clubfoot. J Foot Ankle Surg. 2004; 43:263-65.

14. DiMaggio C, Sun LS, Li G (2011) Early childhood exposure to anesthesia and risk of developmental and behavioral disorders in a sibling birth cohort. Anesth Analg 113(5):1143-51. 


\section{AUTHORSHIP AND CONTRIBUTION DECLARATION}

\begin{tabular}{|c|l|l|l|}
\hline Sr. \# & Author-s Full Name & \multicolumn{1}{|c|}{ Contribution to the paper } \\
\hline 1 & Abdul Latif Shahid & $\begin{array}{l}\text { Pricipal author, Proposal topic, Basic } \\
\text { study design, data collection. } \\
\text { Co-author, methodology, leterature } \\
\text { review. } \\
\text { Co-author, statistical analysis and } \\
\text { interperetation of resutls, Proof } \\
\text { reading + references. }\end{array}$ \\
\hline 3 & Abdul Latif Sami
\end{tabular}

